\title{
Correction to "Translational Pharmacokinetic-Pharmacodynamic Modeling for an Orally Available Novel Inhibitor of Epigenetic Regulator Enhancer of Zeste Homolog 2"
}

The above article [Yamazaki S, Gukasyan HJ, Wang H, Uryu S, and Sharma S (2020) $J$ Pharmacol Exp Ther, 373: 220-229; DOI: https://doi.org/10.1124/jpet.119.263491] was published with an error introduced by the compositor in eqs. 2, 3, 5, 6, and 7. Several of the multiplication symbols were erroneously replaced with $\underset{n}{\ddot{A}}$.

In addition, the authors discovered a typographical error in the compound name PF06821497 in the legends for Figs. 3, 4, and 5 and in the supplemental material.

The PDF and HTML versions of the article and the PDF for the supplemental material have been corrected.

The compositor and the authors apologize for any inconvenience caused by these errors. 